\title{
Implementing Multidimensional Data Warehouses into NoSQL
}

\author{
Max Chevalier ${ }^{1}$, Mohammed El Malki $^{1,2}$, Arlind Kopliku ${ }^{1}$, Olivier Teste ${ }^{1}$ and Ronan Tournier ${ }^{1}$ \\ ${ }^{1}$ University of Toulouse, IRIT UMR 5505 (www.irit.fr), Toulouse, France \\ ${ }^{2}$ Capgemini (www.capgemini.com), Toulouse, France \\ \{Max.Chevalier, Mohammed.ElMalki, Arlind.Kopliku, Olivier.Teste, Ronan.Tournier\}@irit.fr
}

Keywords: NoSQL, OLAP, Aggregate Lattice, Column-Oriented, Document-Oriented.

\begin{abstract}
Not only SQL (NoSQL) databases are becoming increasingly popular and have some interesting strengths such as scalability and flexibility. In this paper, we investigate on the use of NoSQL systems for implementing OLAP (On-Line Analytical Processing) systems. More precisely, we are interested in instantiating OLAP systems (from the conceptual level to the logical level) and instantiating an aggregation lattice (optimization). We define a set of rules to map star schemas into two NoSQL models: columnoriented and document-oriented. The experimental part is carried out using the reference benchmark TPC. Our experiments show that our rules can effectively instantiate such systems (star schema and lattice). We also analyze differences between the two NoSQL systems considered. In our experiments, HBase (columnoriented) happens to be faster than MongoDB (document-oriented) in terms of loading time.
\end{abstract}

\section{INTRODUCTION}

Nowadays, analysis data volumes are reaching critical sizes (Jacobs, 2009) challenging traditional data warehousing approaches. Current implemented solutions are mainly based on relational databases (using R-OLAP approaches) that are no longer adapted to these data volumes (Stonebraker, 2012), (Cuzzocrea et al., 2013), (Dehdouh et al., 2014). With the rise of large Web platforms (e.g. Google, Facebook, Twitter, Amazon, etc.) solutions for "Big Data" management have been developed. These are based on decentralized approaches managing large data amounts and have contributed to developing "Not only SQL" (NoSQL) data management systems (Stonebraker, 2012). NoSQL solutions allow us to consider new approaches for data warehousing, especially from the multidimensional data management point of view. This is the scope of this paper.

In this paper, we investigate the use of NoSQL models for decision support systems. Until now (and to our knowledge), there are no mapping rules that transform a multi-dimensional conceptual model into NoSQL logical models. Existing research instantiate OLAP systems in NoSQL through ROLAP systems; i.e., using an intermediate relational logical model. In this paper, we define a set of rules to translate automatically and directly a conceptual multidimensional model into NoSQL logical models. We consider two NoSQL logical models: one column-oriented and one document-oriented. For each model, we define mapping rules translating from the conceptual level to the logical one. In Figure 1, we position our approach based on abstraction levels of information systems. The conceptual level consists in describing the data in a generic way regardless of information technologies whereas the logical level consists in using a specific technique for implementing the conceptual level.

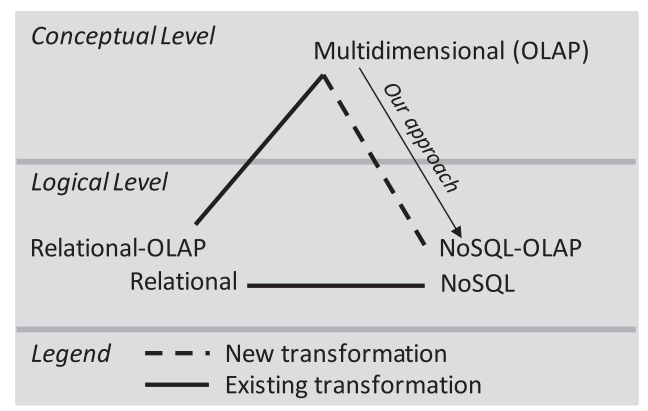

Figure 1: Translations of a conceptual multidimensional model into logical models.

Our motivation is multiple. Implementing OLAP systems using NoSQL systems is a relatively new 
alternative. It is justified by advantages of these systems such as flexibility and scalability. The increasing research in this direction demands for formalization, common models and empirical evaluation of different NoSQL systems. In this scope, this work contributes to investigate two logical models and their respective mapping rules. We also investigate data loading issues including pre-computing data aggregates.

Traditionally, decision support systems use data warehouses to centralize data in a uniform fashion (Kimball et al., 2013). Within data warehouses, interactive data analysis and exploration is performed using On-Line Analytical Processing (OLAP) (Colliat, 1996), (Chaudhuri et al., 1997). Data is often described using a conceptual multidimensional model, such as a star schema (Chaudhuri et al., 1997). We illustrate this multidimensional model with a case study about RSS (Really Simple Syndication) feeds of news bulletins from an information website. We study the Content of news bulletins (the subject of the analysis or fact) using three dimensions of those bulletins (analysis axes of the fact): Keyword (contained in the bulletin), Time (publication date) and Location (geographical region concerned by the news). The fact has two measures (analysis indicators):

- The number of news bulletins (NewsCount).

- The number of keyword occurrences (OccurrenceCount).
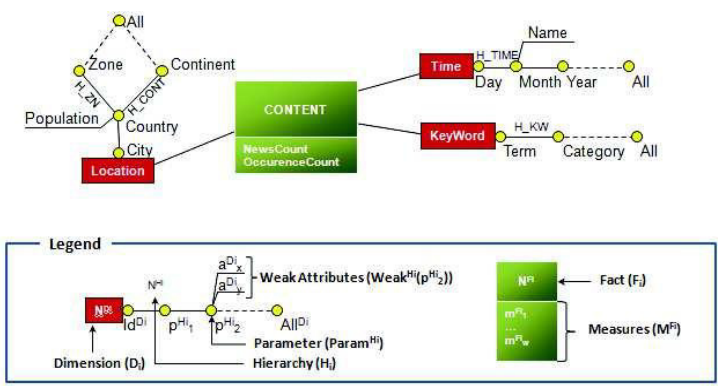

Figure 2: Multidimensional conceptual schema of our example, news bulletin contents according to keywords, publication time and location concerned by the news.

The conceptual multidimensional schema of our case study is described in Figure 2, using a graphical formalism based on (Golfarelli et al., 1998), (Ravat et al., 2008).

One of the most successful implementation of OLAP systems uses relational databases (R-OLAP implementations). In these implementations, the conceptual schema is transformed into a logical schema (i.e. a relational schema, called in this case a denormalized R-OLAP schema) using two transformation rules:

- Each dimension is a table that uses the same name. Table attributes are derived from attributes of the dimension (called parameters and weak attributes). The root parameter is the primary key.

- Each fact is a table that uses the same name, with attributes derived from 1) fact attributes (called measures) and 2) the root parameter of each associated dimension. Attributes derived from root parameters are foreign keys linking each dimension table to the fact table and form a compound primary key of the table.

Due to the huge amount of data that can be stored in OLAP systems, it is common to precompute some aggregated data to speed up common analysis queries. In this case, fact measures are aggregated using different combinations of either dimension attributes or root parameters only. This pre-computation is a lattice of pre-computed aggregates (Gray et al., 1997) or aggregate lattice for short. The lattice is a set of nodes, one per dimension combinations. Each node (e.g. the node called "Time, Location") is stored as a relation called an aggregate relation (e.g. the relation timelocation). This relation is composed of attributes corresponding to the measures and the parameters or weak attributes from selected dimensions. Attributes corresponding to measures are used to store aggregated values computed with functions such as SUM, COUNT, MAX, MIN, etc. The aggregate lattice schema for our case study is shown in Figure 3.

Considering NoSQL systems as candidates for implementing OLAP systems, we must consider the above issues. In this paper and, in order to deal with these issues, we use two logical NoSQL models for the logical implementation; we define mapping rules (that allows us to translate a conceptual design into a logical one) and we study the lattice computation.

The rest of this paper is organized as follows: in section 2, we present possible approaches that allow getting a NoSQL implementation from a data warehouse conceptual model using a pivot logical model; in section 3 we define our conceptual multidimensional model, followed by a section for each of the two NoSQL models we consider along with their associated transformation rules, i.e. the column-oriented model in section 4 and the document-oriented model in section 5. Finally, section 6 details our experiments. 


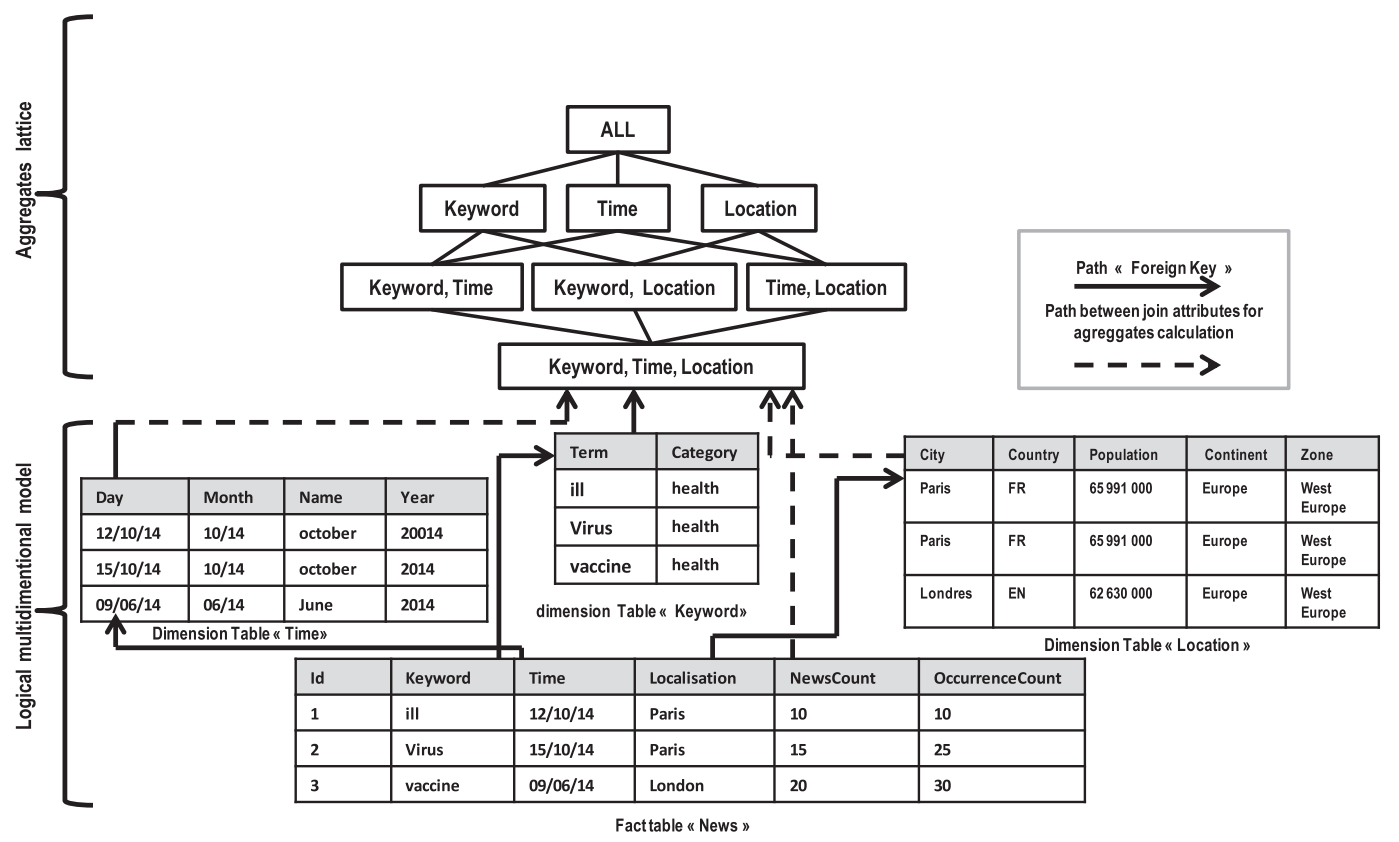

Figure 3: A pre-computed aggregate lattice in a R-OLAP system.

\section{RELATED WORK}

To our knowledge, there is no work for automatically and directly transforming data warehouses defined by a multidimensional conceptual model into a NoSQL model.

Several research works have been conducted to translate data warehousing concepts to a relational R-OLAP logical level (Morfonios et al., 2007). Multidimensional databases are mostly implemented using these relational technologies. Mapping rules are used to convert structures of the conceptual level (facts, dimensions and hierarchies) into a logical model based on relations. Moreover, many works have focused on implementing logical optimization methods based on pre-computed aggregates (also called materialized views) as in (Gray et al., 1997), (Morfonios et al., 2007). However, R-OLAP implementations suffer from scaling-up to large data volumes (i.e. "Big Data"). Research is currently under way for new solutions such as using NoSQL systems (Lee et al., 2012). Our approach aims at revisiting these processes for automatically implementing multidimensional conceptual models directly into NoSQL models.

Other studies investigate the process of transforming relational databases into a NoSQL logical model (bottom part of Figure 1). In ( $\mathrm{Li}$, 2010), the author has proposed an approach for transforming a relational database into a columnoriented NoSQL database using HBase (Han et al., 2012), a column-oriented NoSQL database. In (Vajk et al., 2013), an algorithm is introduced for mapping a relational schema to a NoSQL schema in MongoDB (Dede et al., 2013), a document-oriented NoSQL database. However, these approaches never consider the conceptual model of data warehouses. They are limited to the logical level, i.e. transforming a relational model into a columnoriented model. More specifically, the duality fact/dimension requires guaranteeing a number of constraints usually handled by the relational integrity constraints and these constraints cannot be considered in these logical approaches.

This study highlights that there is currently no approaches for automatically and directly transforming a data warehouse multidimensional conceptual model into a NoSQL logical model. It is possible to transform multidimensional conceptual models into a logical relational model, and then to transform this relational model into a logical NoSQL model. However, this transformation using the relational model as a pivot model has not been formalized as both transformations were studied independently of each other. Also, this indirect approach can be tedious. 
We can also cite several recent works that are aimed at developing data warehouses in NoSQL systems whether columns-oriented (Dehdouh et al., 2014), or key-values oriented (Zhao et al., 2014). However, the main goal of these papers is to propose benchmarks. These studies have not put the focus on the model transformation process. Likewise, they only focus one NoSQL model, and limit themselves to an abstraction at the HBase logical level. Both models (Dehdouh et al., 2014), (Zhao et al., 2014), require the relational model to be generated first before the abstraction step. By contrast, we consider the conceptual model as well as two orthogonal logical models that allow distributing multidimensional data either vertically using a column-oriented model or horizontally using a document-oriented model.

Finally we take into account hierarchies in our transformation rules by providing transformation rules to manage the aggregate lattice.

\section{CONCEPTUAL MULTI- DIMENSIONAL MODEL}

To ensure robust translation rules we first define the multidimensional model used at the conceptual level.

A multidimensional schema, namely $E$, is defined by $\left(F^{E}, D^{E}, \operatorname{Star}^{E}\right)$ where:

- $F^{E}=\left\{F_{1}, \ldots, F_{n}\right\}$ is a finite set of facts,

- $D^{E}=\left\{D_{1}, \ldots, D_{m}\right\}$ is a finite set of dimensions,

- $\operatorname{Star}^{E}: F^{E} \rightarrow 2^{D^{E}}$ is a function that associates each fact $F_{i}$ of $F^{E}$ to a set of $D_{i}$ dimensions, $D_{i} \in \operatorname{Star}^{E}\left(F_{i}\right)$, along which it can be analyzed; note that $2^{D^{E}}$ is the power set of $D^{E}$.

A dimension, denoted $D_{i} \in D^{E}$ (abusively noted as $D)$, is defined by $\left(N^{D}, A^{D}, H^{D}\right)$ where:

- $\quad N^{D}$ is the name of the dimension,

- $A^{D}=\left\{a_{1}^{D}, \ldots, a_{u}^{D}\right\} \cup\left\{i d^{D}, A l l^{D}\right\}$ is a set of dimension attributes,

- $H^{D}=\left\{H_{1}^{D}, \ldots, H_{v}^{D}\right\}$ is a set hierarchies.

A hierarchy of the dimension $D$, denoted $H_{i} \in H^{D}$, is defined by $\left(N^{H i}, \operatorname{Param}^{H i}\right.$, Weak $\left.{ }^{H i}\right)$ where:

- $N^{H i}$ is the name of the hierarchy,

- $\operatorname{Param}_{i}^{H_{i}}=<i d^{D}, p_{1}^{H_{i}}, \ldots, p_{v_{i}}^{H_{i}}, A l l^{D}>$ is an ordered set of $v_{i}+2$ attributes which are called parameters of the relevant graduation scale of the hierarchy, $\forall k \in\left[1 . . v_{\mathrm{i}}\right], p_{k}^{H i} \in A^{D}$.

- Weak ${ }^{H i}{\text { : } \text { Param }^{H i}}_{\rightarrow} 2^{A^{D}-\text { Param }^{H i}}$ is a function associating with each parameter zero or more weak attributes.
A fact, $F \in F^{E}$, is defined by $\left(N^{F}, M^{F}\right)$ where:

- $N^{F}$ is the name of the fact,

- $M^{F}=\left\{f_{1}\left(m_{1}^{F}\right), \ldots, f_{v}\left(m_{v}^{F}\right)\right\}$ is a set of measures, each associated with an aggregation function $f_{i}$.

Example. Consider our case study where news bulletins are loaded into a multidimensional data warehouse consistent with the conceptual schema described in Figure 2.

The multidimensional schema $E^{\text {News }}$ is defined by $F^{\text {News }}=\left\{F_{\text {Content }}\right\}, D^{\text {News }}=\left\{D_{\text {Time }}, D_{\text {Location }}, D_{\text {Keyword }}\right\}$ and $\operatorname{Star}^{\text {News }}\left(F_{\text {Content }}\right)=\left\{D_{\text {Time }}, D_{\text {Location }}, D_{\text {Keyword }}\right\}$.

The fact represents the data analysis of the news feeds and uses two measures: the number of news (NewsCount) and the number of occurrences (OccurrenceCount); both for the set of news corresponding to a given term (or keyword), a specific date and a given location. This fact, $F_{\text {Content }}$ is defined by (Content, $\{S U M$ (NewsCount), $\operatorname{SUM}($ OccurrenceCount $)\})$ and is analyzed according to three dimensions, each consisting of several hierarchical levels (detail levels):

- The geographical location (Location) concerned by the news (with levels City, Country, Continent and Zone). A complementary information of the country being its Population (modeled as additional information; it is a weak attribute).

- The publication date (Time) of the bulletin (with levels Day, Month and Year); note that the month number is associated to its Name (also a weak attribute),

- The Keyword used in the News (with the levels Term and Category of the term).

For instance, the dimension $D_{\text {Location }}$ is defined by (Location, \{City, Country, Continent, Zone, $\left.\left.A L L^{\text {Location }}\right\},\left\{H_{C o n t}, H_{Z n}\right\}\right)$ with City $=i d^{\text {Location }}$ and:

- $H_{\text {Cont }}=$ (HCont, \{City, Country, Continent, ALL $L^{\text {Location }}$ \}, (Country, \{Population $\left.\left.\}\right)\right) ;$ note that Weak $k^{\text {HCont }}($ Country $)=\{$ Population $\}$,

- $H_{Z n}=(H Z n,\{$ City, Country, Zone, ALL Location $\}$, (Country, \{Population $\})$ ).

\section{CONVERSION INTO A NOSQL COLUMN-ORIENTED MODEL}

The column-oriented model considers each record as a key associated with a value decomposed in several columns. Data is a set of lines in a table composed of columns (grouped in families) that may be different from one row to the other. 


\subsection{NoSQL Column-Oriented Model}

In relational databases, the data structure is determined in advance with a limited number of typed columns (a few thousand) each similar for all records (also called "tuples"). Column-oriented NoSQL models provide a flexible schema (untyped columns) where the number of columns may vary between each record (or "row").

A column-oriented database (represented in Figure 4) is a set of tables that are defined row by row (but whose physical storage is organized by groups of columns: column families; hence a "vertical partitioning" of the data). In short, in these systems, each table is a logical mapping of rows and their column families. A column family can contain a very large number of columns. For each row, a column exists if it contains a value.

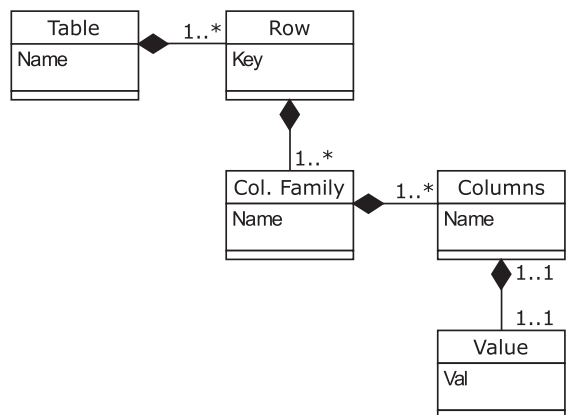

Figure 4: UML class diagram representing the concepts of a column-oriented NoSQL database (tables, rows, column families and columns).

A table $T=\left\{R_{1}, \ldots, R_{n}\right\}$ is a set of rows $R_{i}$. A row $R_{i}=\left(\right.$ Key $\left._{i},\left(C F_{i}{ }^{1}, \ldots, C F_{i}^{m}\right)\right)$ is composed of a row key $K e y_{i}$ and a set of column families $C F_{i}^{j}$.

A column family $C F_{i}^{j}=\left\{\left(C_{i}^{j 1},\left\{v_{i}^{j 1}\right\}\right), \ldots,\left(C_{i}^{j p}\right.\right.$, $\left.\left.\left\{v_{i}^{j p}\right\}\right)\right\}$ consists of a set of columns, each associated with an atomic value. Every value can be "historised" thanks to a timestamp. This principle useful for version management (Wrembel, 2009) will not be used in this paper due to limited space, although it may be important.

The flexibility of a column-oriented NoSQL database allows managing the absence of some columns between the different table rows. However, in the context of multidimensional data storage, data is usually highly structured (Malinowski et al., 2006). Thus, this implies that the structure of a column family (i.e. the set of columns defined by the column family) will be the same for all the table rows. The initial structure is provided by the data integration process called ETL, Extract, Transform, and Load (Simitsis et al., 2005).
Example: Let us have a table $T^{\text {News }}$ representing aggregated data related to news bulletins (see Figure 5), with: $T^{\text {News }}=\left\{R_{1}, \ldots, R_{x}, \ldots, R_{n}\right\}$. We detail the $R_{x}$ row that corresponds to the number of news bulletins, and the number of occurrences where the keyword "Iraq" appears in those news bulletins, published at the date of 09/22/2014 and concerning the location "Toulouse" (south of France).

$R_{x}=\left(x, \quad\left(C F_{x}{ }^{\text {Time }}=\left\{\left(C_{x}^{\text {Day }},\left\{V_{x}^{\text {Day }}\right\}\right), \quad\left(C_{x}^{\text {Month }}, V_{x}^{\text {Month }}\right)\right.\right.\right.$, $\left.\left(C_{x}^{\text {Name }}, V_{x}^{\text {Name }}\right),\left(C_{x}^{\text {Year }}, V_{x}^{\text {Year }}\right)\right\}, C F_{x}^{\text {Location }}=\left\{\left(C_{x}^{\text {City }}\right.\right.$, $\left.V_{x}^{\text {City }}\right),\left(C_{x}^{\text {Country }}, V_{x}^{\text {Country }}\right),\left(C_{x}^{\text {Population }}, V_{x}^{\text {Population }}\right)$, $\left.\left(C_{x}^{\text {Continent }}, V_{x}^{\text {Continent }}\right),\left(C_{x}^{\text {Zone }}, V_{x}^{\text {Zone }}\right)\right\}, \quad C F_{x}^{\text {Keyword }}$ $=\left\{\left(C_{x}^{\text {Term }}, V_{x}^{\text {Term }}\right), \quad\left(C_{x}^{\text {Category }}, V_{x}^{\text {Category }}\right)\right\}, \quad C F_{x}^{\text {Content }}$ $=\left\{\left(C_{x}^{\text {NewsCount }}, \quad V_{x}^{\text {NewsCount }}\right), \quad\left(C_{x}^{\text {OccurrenceCount }}\right.\right.$, $\left.\left.\left.V_{x}^{\text {OccurrenceCount }}\right)\right\}\right)$ )

The values of the five columns of $C F_{x}^{\text {Location, }}$ $\left(C_{x}^{\text {City }}, C_{x}^{\text {Country }}, C_{x}^{\text {Population }}, C_{x}^{\text {Continent }}\right.$ and $\left.C_{x}^{\text {Zone }}\right)$, are $\left(V_{x}^{\text {City }}, V_{x}^{\text {Country }}, V_{x}^{\text {Population }}, V_{x}^{\text {Continent }}\right.$ and $\left.V_{x}^{\text {Zone }}\right)$; e.g. $V_{x}^{\text {City }}=$ Toulouse, $V_{x}^{\text {Country }}=$ France, $V_{x}^{\text {Population }}=$ $65991000, V_{x}^{\text {Continent }}=$ Europe, $V_{x}^{\text {Zone }}=$ EuropeWestern.

More simply we note: $C F_{x}^{\text {Location }}=\{($ City, $\{$ Toulouse $\}), \quad$ Country, $\{$ France $\}), \quad$ (Population, \{65991000\}), (Continent, \{Europe\}), (Zone, \{Europe-Western $\}$ ) .

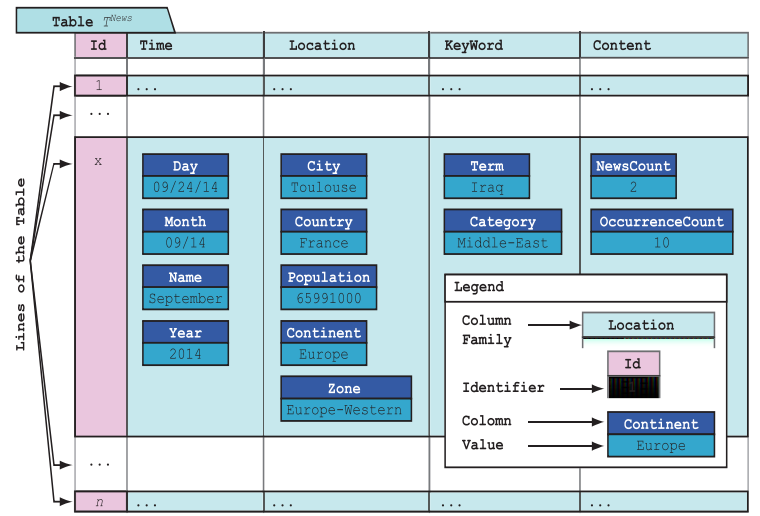

Figure 5: Example of a row $(\operatorname{key}=x)$ in the $T^{\text {News }}$ table.

\subsection{Column-Oriented Model Mapping Rules}

The elements (facts, dimensions, etc.) of the conceptual multidimensional model have to be transformed into different elements of the columnoriented NoSQL model (see Figure 6).

- Each conceptual star schema (one $F_{i}$ and its associated dimensions $\left.\operatorname{Star}^{E}\left(F_{i}\right)\right)$ is transformed into a table $T$.

- The fact $F_{i}$ is transformed into a column family $C F^{M}$ of $T$ in which each measure $m_{i}$ is a column $C_{i} \in C F^{M}$. 
- Each dimension $D_{i} \in \operatorname{Star}^{E}\left(F^{i}\right)$ is transformed into a column family $C F^{D i}$ where each dimension attribute $A_{i} \in A^{D}$ (parameters and weak attributes) is transformed into a column $C_{i}$ of the column family $C F^{D i}\left(C_{i} \in C F^{D i}\right)$, except the parameter $A l l^{D i}$.

Remarks. Each fact instance and its associated instances of dimensions are transformed into a row $R_{x}$ of $T$. The fact instance is thus composed of the column family $C F^{M}$ (the measures and their values) and the column families of the dimensions $C F^{D i} \in$ $C F^{D E}$ (the attributes, i.e. parameters and weak attributes, of each dimension and their values).

As in a denormalized R-OLAP star schema (Kimball et al., 2013), the hierarchical organization of the attributes of each dimension is not represented in the NoSQL system. Nevertheless, hierarchies are used to build the aggregate lattice. Note that the hierarchies may also be used by the ETL processes which build the instances respecting the constraints induced by these conceptual structures (Malinowski et al., 2006); however, we do not consider ETL processes in this paper.

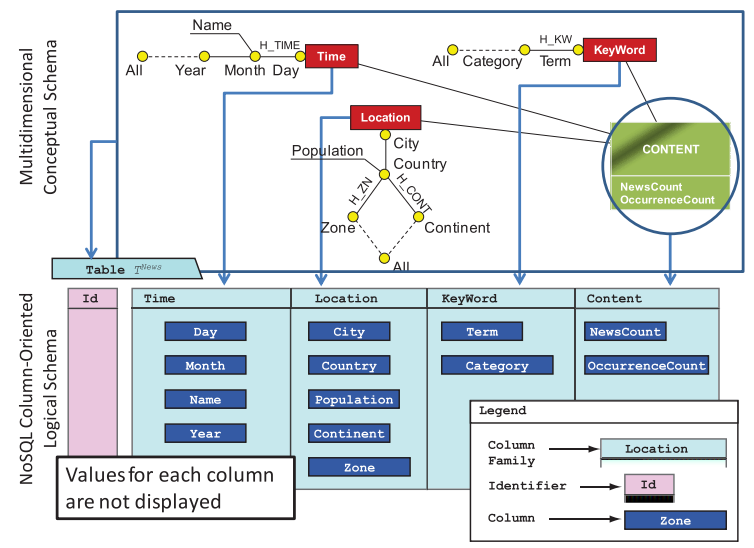

Figure 6: Implementing a multidimensional conceptual model into the column-oriented NoSQL logical model.

Example. Let $E^{\text {News }}$ be the multidimensional conceptual schema implemented using a table named $T^{\text {News }}$ (see Figure 6$)$. The fact $\left(F^{\text {Contents }}\right)$ and its dimensions $\left(D^{\text {Time }}, D^{\text {Localisation }}, D^{\text {Keyword }}\right)$ are implemented into four column families $C F^{\text {Time }}$, $C F^{\text {Location }}, C F^{\text {Keyword }}, C F^{\text {Contents }}$. Each column family contains a set of columns, corresponding either to dimension attributes or to measures of the fact. For instance the column family $C F^{\text {Location }}$ is composed of the columns $\left\{C^{\text {City }}, C^{\text {Country }}, C^{\text {Population }}, C^{\text {Continent }}\right.$, $C^{\text {Zone }}$.

Unlike R-OLAP implementations, where each fact is translated into a central table associated with dimension tables, our rules translate the schema into a single table that includes the fact and its associated dimensions together. When performing queries, this approach has the advantage of avoiding joins between fact and dimension tables. As a consequence, our approach increases information redundancy as dimension data is duplicated for each fact instance. This redundancy generates an increased volume of the overall data while providing a reduced query time. In a NoSQL context, problems linked to this volume increase may be reduced by an adapted data distribution strategy. Moreover, our choice for accepting this important redundancy is motivated by data warehousing context where data updates consist essentially in inserting new data; additional costs incurred by data changes are thus limited in our context.

\subsection{Lattice Mapping Rules}

We will use the following notations to define our lattice mapping rules. A pre-computed aggregate lattice or aggregate lattice $L$ is a set of nodes $A^{L}$ (pre-computed aggregates or aggregates) linked by edges $E^{L}$ (possible paths to calculate the aggregates). An aggregate node $A \in A^{L}$ is composed of a set of $p_{i}$ parameters (one by dimension) and a set of aggregated $m_{i}$ measures $f_{i}\left(m_{i}\right) . A=<p_{1} \ldots p_{k}$, $f_{1}\left(m_{1}\right), \ldots, f_{v}\left(m_{v}\right)>, k \leq m$ ( $m$ being the number of dimensions, $v$ being the number of measures of the fact).

The lattice can be implemented in a columnoriented NoSQL database using the following rules:

- Each aggregate node $A \in A^{L}$ is stored in a dedicated table.

- For each dimension $D_{i}$ associated to this node, a column family $C F^{D i}$ is created, each dimension attribute $a_{i}$ of this dimension is stored in a column $C$ of $C F^{D i}$,

- The set of aggregated measures is also stored in a column family $C F^{F}$ where each aggregated measure is stored as a column $C$ (see Figure 7).

Example. We consider the lattice News (see Figure 7). The lattice News is stored in tables. The node (Keyword_Time) is stored in a Table $T^{\text {Keyword_Time }}$ composed of the column families $C F^{\text {Keyword }}, C F^{\text {Time }}$ and $C F^{\text {Fact }}$. The attribute Year is stored in a column $C^{\text {Year }}$, itself in $C F^{\text {Time }}$. The attribute Term is stored in a column $C^{\text {Term }}$, itself in $C F^{\text {Keyword }}$. The two measures are stored as two columns in the column family $C F^{\text {fact }}$. 
Many studies have been conducted about how to select the pre-computed aggregates that should be computed. In our proposition we favor computing all aggregates (Kimball et al., 2013). This choice may be sensitive due to the increase in the data volume. However, in a NoSQL architecture we consider that storage space should not be a major issue.

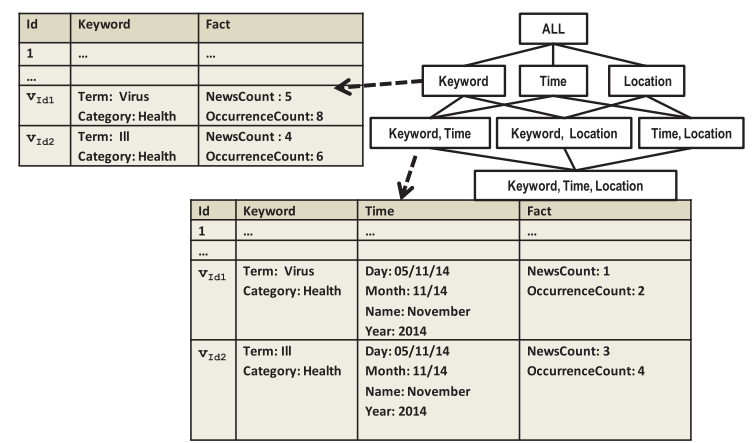

Figure 7: Implementing the pre-computed aggregation lattice into a column-oriented NoSQL logical model.

\section{CONVERSION INTO A NOSQL DOCUMENT-ORIENTED MODEL}

The document-oriented model considers each record as a document, which means a set of records containing "attribute/value" pairs; these values are either atomic or complex (embedded in sub-records). Each sub-record can be assimilated as a document, i.e. a subdocument.

\subsection{NoSQL Document-Oriented Model}

In the document-oriented model, each key is associated with a value structured as a document. These documents are grouped into collections. A document is a hierarchy of elements which may be either atomic values or documents. In the NoSQL approach, the schema of documents is not established in advance (hence the "schema less" concept).

Formally, a NoSQL document-oriented database can be defined as a collection $C$ composed of a set of documents $D_{i}, C=\left\{D_{1}, \ldots, D_{n}\right\}$.

Each $D_{i}$ document is defined by a set of pairs $D_{i}=\left\{\left(A t t_{i}^{1}, V_{i}^{1}\right), \ldots,\left(A t t_{i}^{m}, V_{i}^{m}\right)\right\}, j \in[1, m]$ where $A t t_{i}^{j}$ is an attribute (which is similar to a key) and $V_{i}^{j}$ is a value that can be of two forms:

- The value is atomic.
- The value is itself composed by a nested document that is defined as a new set of pairs (attribute, value).

We distinguish simple attributes whose values are atomic from compound attributes whose values are documents called nested documents.

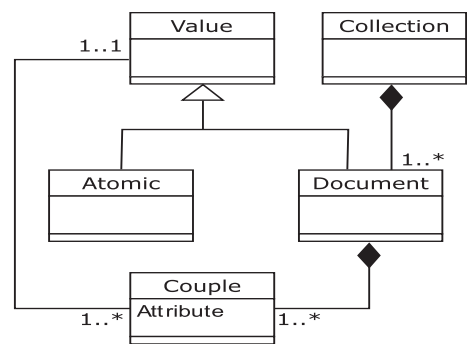

Figure 8: UML class diagram representing the concepts of a document-oriented NoSQL database.

Example. Let $C$ be a collection, $C=\left\{D_{1}, \ldots, D_{x}, \ldots, D_{n}\right\} \quad$ in which we detail the document $D_{x}$ (see Figure 9). Suppose that $D_{x}$ provides the number of news and the number of occurrences for the keyword "Iraq" in the news having a publication date equals to $09 / 22 / 2014$ and that are related to Toulouse.

Within the collection $C^{\text {News }}=\left\{D_{1}, \ldots, D_{x}, \ldots, D_{n}\right\}$, the document $D_{x}$ could be defined as follows:

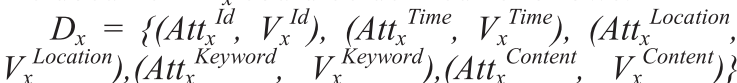
where $A t t_{x}^{I d}$ is a simple attribute and while the other $4\left(\right.$ Att $_{x}^{\text {Time }}, \mathrm{Att}_{x}^{\text {Location }}, \mathrm{Att}_{x}^{\text {Keyword }}$, and $\left.\mathrm{Att}_{x}^{\text {Content }}\right)$ are compound attributes. Thus, $V_{x}^{I d}$ is an atomic value (e.g. " $X$ ") corresponding to the key (that has to be unique). The other 4 values $\left(V_{x}^{\text {Time }}, V_{x}^{\text {Location }}\right.$, $V_{x}^{\text {Keyword }}$, and $\left.V_{x}^{\text {Content }}\right)$ are nested documents:

$$
\begin{aligned}
& V_{x}^{\text {Time }}=\left\{\left(A t t_{x}^{\text {Day }}, V_{x}^{\text {Day }}\right),\left(A t t_{x}^{\text {Month }}, V_{x}^{\text {Month }}\right)\right. \text {, } \\
& V_{x}^{\text {Location }}=\left\{\left(\text { Att }_{x}^{\text {City }}, V_{x}^{\text {City }}\right),\left(\text { Att }_{x}^{\text {Country }}, V_{x}^{\text {Country }}\right),\right. \\
& \left(\text { Att } t_{x}^{\text {Population }}, V_{x}^{\text {Population }}\right) \text {, (Att }{ }_{x}^{\text {Continent }} \text {, } \\
& V_{x}^{\text {Keyword }}=\left\{\left(\text { Att }_{x}^{\text {Term }}, V_{x}^{V_{x}^{\text {Term }}}\right),\right. \\
& \text { (Att } \left.\left.x_{x}^{\text {Category }}, V_{x}^{\text {Category }}\right)\right\} \text {, } \\
& V_{x}^{\text {Contents }}=\left\{\left(\text { Att }_{x}^{\text {NewsCount }}, V_{x}^{\text {NewsCount }}\right),\right. \\
& \text { (Att } \left.\left.{ }_{x}^{\text {OccurenceCount }}, V_{x}^{\text {OccurenceCount }}\right)\right\} \text {. }
\end{aligned}
$$

In this example, the values in the nested documents are all atomic values. For example, values associated to the attributes Att $_{x}^{\text {City }}, A^{\text {Att }}{ }^{\text {Country, }}$, $A t t_{x}^{\text {Population }}, \mathrm{Att}_{x}^{\text {Continent }}$ and $A t t_{x}^{\text {Zone }}$ are:

$$
\begin{aligned}
& V_{x}^{\text {City }}=\text { "Toulouse", } \\
& V_{x}^{\text {Country }}=\text { "France", } \\
& V_{x}^{\text {Population }}=\text { "65991000", } \\
& V_{x}^{\text {Continent }}=\text { "Europe", } \\
& V_{x}^{\text {Zone }}=\text { "Europe Western". }
\end{aligned}
$$


See Figure 9 for the complete example.

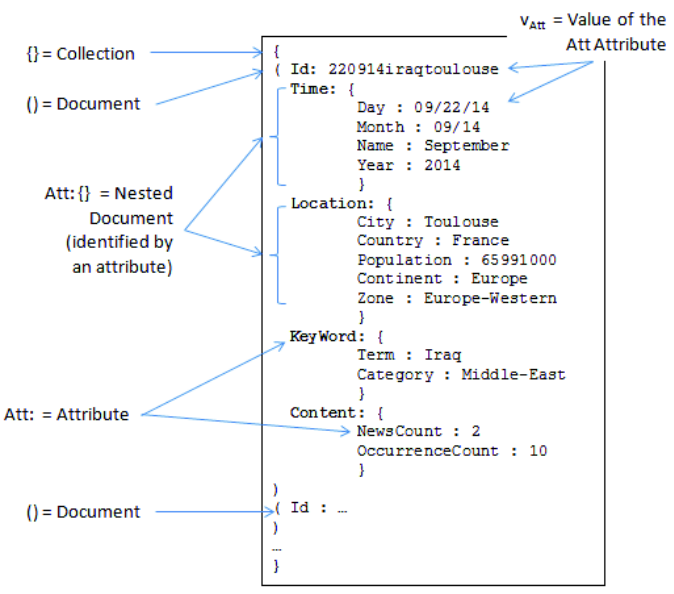

Figure 9: Graphic representation of a collection $C^{\text {News }}$.

\subsection{Document-Oriented Model Mapping Rules}

Under the NoSQL document-oriented model, the data is not organized in rows and columns as in the previous model, but it is organized in nested documents (see Figure 10).

- Each conceptual star schema (one $F_{i}$ and its dimensions $\operatorname{Star}^{E}\left(F_{i}\right)$ ) is translated in a collection $C$.

- The fact $F_{i}$ is translated in a compound attribute $A t t^{C F}$. Each measure $m_{i}$ is translated into a simple attribute $A t t^{S M}$.

- Each dimension $D_{i} \in \operatorname{Star}^{E}\left(F_{i}\right)$ is converted into a compound attribute $A t t^{C D}$ (i.e. a nested document). Each attribute $A_{i} \in A^{D}$ (parameters and weak attributes) of the dimension $D_{i}$ is converted into a simple attribute $A t t^{A}$ contained in $A t t^{C D}$.

Remarks. A fact instance is converted into a document $d$. Measures values are combined within a nested document of $d$. Each dimension is also translated as a nested document of $d$ (each combining parameter and weak attribute values).

The hierarchical organization of the dimension is not preserved. But as in the previous approach, we use hierarchies to build the aggregate lattice.

Example. The document noted $D_{x}$ is composed of 4 nested documents, Att ${ }^{\text {Content }}$, that groups measures and Att $t^{\text {Location }}, A t t^{\text {Keyword }}, A t t^{\text {Time }}$, that correspond to the instances of each associated dimension.

As the previous model, the transformation process produces a large collection of redundant data. This choice has the advantage of promoting data querying where each fact instance is directly combined with the corresponding dimension instances. The generated volume can be compensated by an architecture that would massively distribute this data.

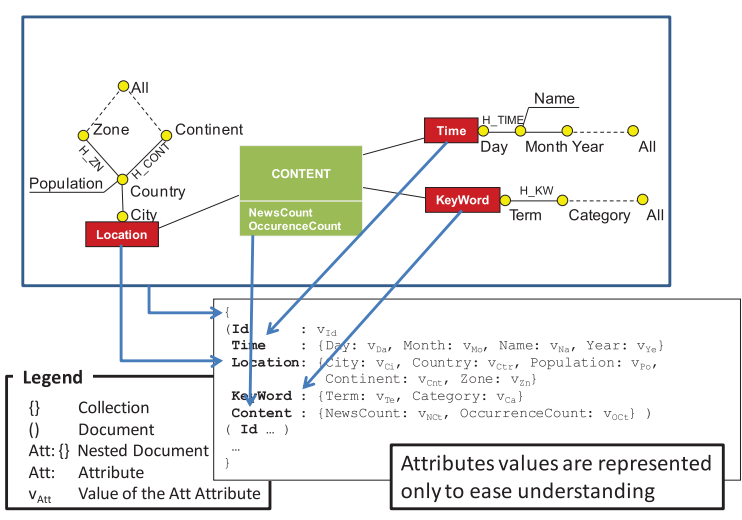

Figure 10: Implementing the conceptual model into a document-oriented NoSQL logical model.

\subsection{Lattice Mapping Rules}

As in the previous approach, we store all the precomputed aggregates in a separate unique collection.

Formally, we use the same definition for the aggregate lattice as above (see section 4.3). However, when using a document oriented NoSQL model, the implementation rules are:

- Each node $A$ is stored in a collection.

- For each dimension $D_{i}$ concerned by this node, a compound attribute (nested document) $A t t_{D i}^{C D}$ is created; each attribute $a_{i}$ of this dimension is stored in a simple attribute $A t t^{a i}$ of $A t t^{C D}$.

- The set of aggregated measures is stored in a compound attribute $A t t_{F}^{C D}$ where each aggregated measure is stored as a simple attribute $A t t_{m i}$

Example. Let us Consider the lattice $L^{\text {News }}$ (see Figure 11). This lattice is stored in a collection $\mathrm{C}^{\text {News }}$. The Node <month_country> is stored as a document $d$. The dimension Time and Location are stored in a nested document $d^{\text {date }}$ and $d^{\text {location }}$ of $d$. The month attribute is stored as a simple attribute in the nested document $d^{\text {Time }}$. The country attribute is stored in a nested document $d^{\text {location }}$ as simple attribute. The two measures are also stored in a nested document denote $d^{\text {fact }}$. 


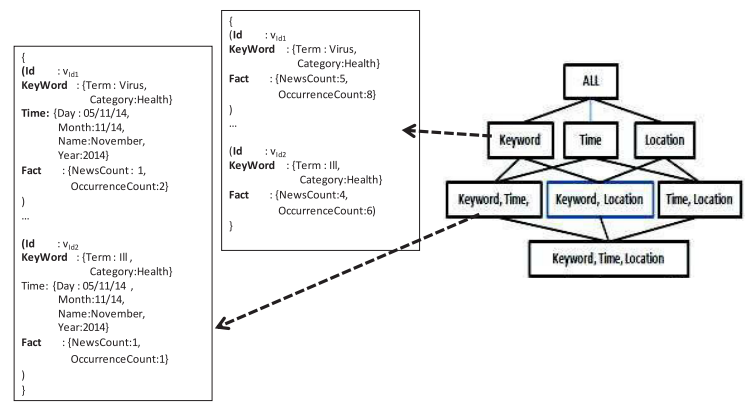

Figure 11: Implementing the pre-computed aggregation lattice into a document-oriented NoSQL logical model.

As in the column-oriented model, we choose to store all possible aggregates of the lattice. With this choice there is a large potential decrease in terms of query response time.

\section{EXPERIMENTS}

\subsection{Experimental setup}

Our experimental goal is to illustrate the instantiation of our logical models (both star schema and lattice levels). Thus, we show here experiments with respect to data loading and lattice generation.

We use HBase and MongoDB respectively for testing the column-oriented and the documentoriented models. Data is generated with a reference benchmark (TPC-DS, 2014). We generate datasets of sizes: $1 \mathrm{~GB}, 10 \mathrm{~GB}$ and 100GB. After loading data, we compute the aggregate lattice with mapreduce/aggregations offered by both HBase and MongoDB. The details of the experimental setup are as follows:

Dataset. The TPC-DS benchmark is used for generating our test data. This is a reference benchmark for testing decision support (including OLAP) systems. It involves a total of 7 fact tables and 17 shared dimension tables. Data is meant to support a retailer decision system. We use the store_sales fact and its 10 associated dimensions tables (the most used ones). Some of its dimensions tables are higher hierarchically organized parts of other dimensions. We consider aggregations on the following dimensions: date (day, month, year), customer address (city, country), store address (city, country) and item (class, category).

Data generation. Data is generated by the DSGen generator (1.3.0). Data is generated in different CSV-like files (Coma Separated Values), one per table (whether dimension or fact). We process this data to keep only the store_sales measures and associated dimension values (by joining across tables and projecting the data). Data is then formatted as CSV files and JSon files, used for loading data in respectively HBase and MongoDB. We obtain successively $1 G B, 10 G B$ and $100 G B$ of random data. The JSon files turn out to be approximately 3 times larger for the same data. The entire process is shown in the Figure 12.

Data loading. Data is loaded into HBase and MongoDB using native instructions. These are supposed to load data faster when loading from files. The current version of MongoDB would not load data with our logical model from CSV file, thus we had to use JSON files.

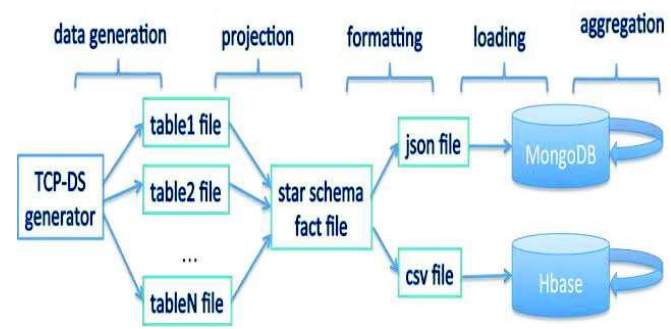

Figure 12: Broad schema of the experimental setup.

Lattice computation. To compute the aggregate lattice, we use map-reduce functions from both HBase and MongoDB. Four levels of aggregates are computed on top of the detailed facts. These aggregates are: all combinations of 3 dimensions, all combinations of 2 dimensions, all combinations of 1 dimension, all data.

MongoDB and HBase allow aggregating data using map-reduce functions which are efficient for distributed data systems. At each aggregation level, we apply aggregation functions: $\max$, min, sum and count on all dimensions. For MongoDB, instructions look like:

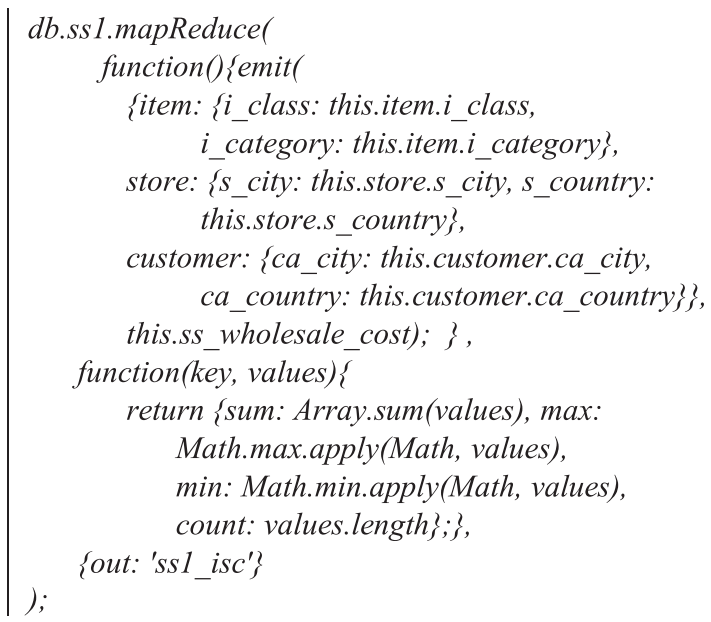




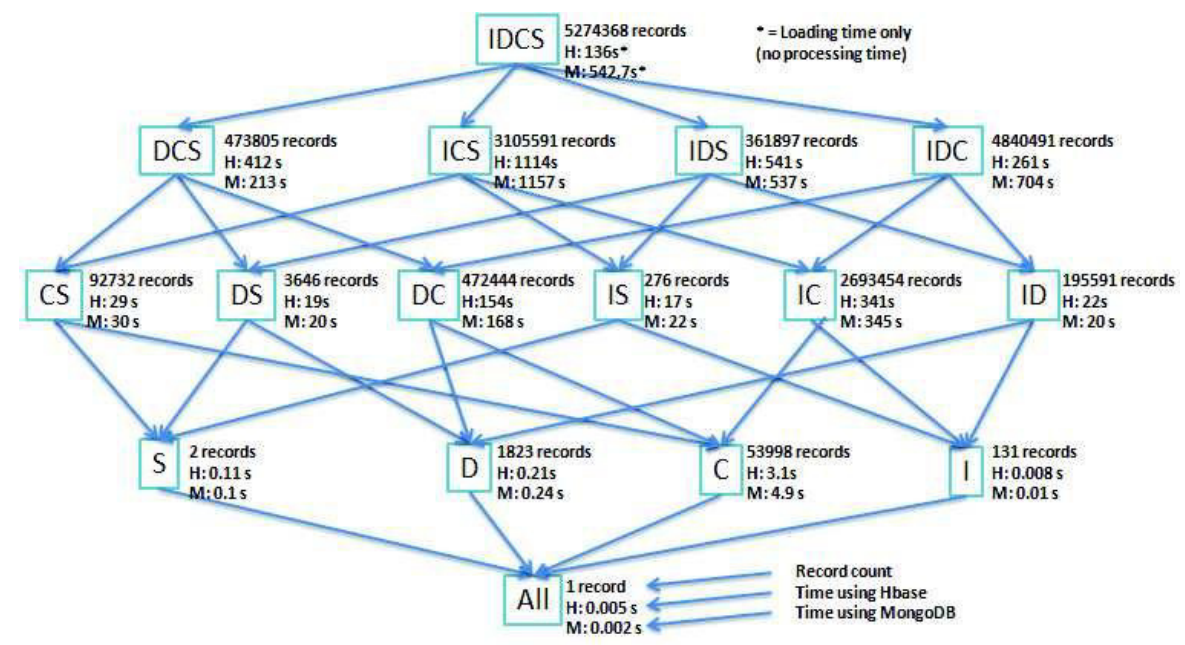

Figure 13: The pre-computed aggregate lattice with processing time (seconds) and size (records/documents), using HBase $(\mathrm{H})$ and MongoDB $(\mathrm{M})$. The dimensions are abbreviated (D: Date, I: item, S: store, C: customer).

In the above, data is aggregated using the item, store and customer dimensions. For HBase, we use Hive on top to ease the query writing for aggregations. Queries with Hive are SQL-like. The below illustrates the aggregation on item, store and customer dimensions.

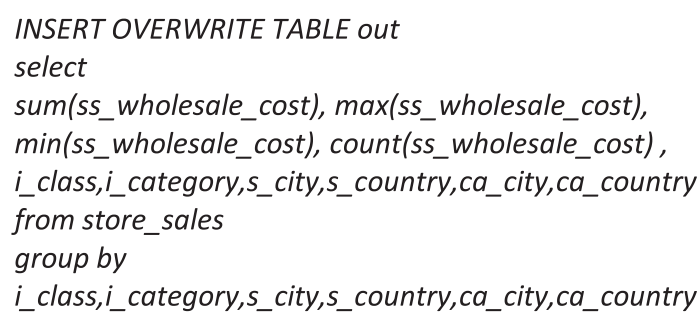

Hardware. The experiments are done on a cluster composed of 3 PCs, (4 core-i5, 8GB RAM, $2 \mathrm{~TB}$ disks, $1 \mathrm{~Gb} / \mathrm{s}$ network), each being a worker node and one node acts also as dispatcher.

Data Management Systems. We use two NoSQL data management systems: HBase (v.0.98) and MongoDB (v.2.6). They are both successful key-value database management systems respectively for column-oriented and documentoriented data storage. Hadoop (v.2.4) is used as the underlying distributed storage system.

\subsection{Experimental results}

Loading data: The data generation process produced files respectively of $1 \mathrm{~GB}, 10 \mathrm{~GB}$, and 100GB. The equivalent files in JSon where about 3.4 times larger due to the extra format. In the table below, we show loading times for each dataset and for both HBase and MongoDB. Data loading was successful in both cases. It confirms that HBase is faster when it comes to loading. However, we did not pay enough attention to tune each system for loading performance. We should also consider that the raw data (JSon files) takes more space in memory in the case of MongoDB for the same number of records. Thus we can expect a higher network transfer penalty.

Table 1: Dataset loading times for each NosQL database management system.

\begin{tabular}{|l|r|r|r|}
\hline Dataset size & \multicolumn{1}{|c|}{ 1GB } & \multicolumn{1}{c|}{ 10GB } & \multicolumn{1}{c|}{ 100GB } \\
\hline MongoDB & $9.045 \mathrm{~m}$ & $109 \mathrm{~m}$ & $132 \mathrm{~m}$ \\
\hline HBase & $2.26 \mathrm{~m}$ & $2.078 \mathrm{~m}$ & $10,3 \mathrm{~m}$ \\
\hline
\end{tabular}

Lattice computation: We report here the experimental observations on the lattice computation. The results are shown in the schema of Figure 13. Dimensions are abbreviated (D: date, C: customer, I: item, S: store). The top level corresponds to IDCS (detailed data). On the second level, we keep combinations of only three dimensions and so on. For every aggregate node, we show the number of records/documents it contains and the computation time in seconds respectively for HBase $(\mathrm{H})$ and MongoDB $(\mathrm{M})$.

In HBase, the total time to compute all aggregates was 1700 seconds with respectively $1207 \mathrm{~s}, 488 \mathrm{~s}, 4 \mathrm{~s}$ and $0.004 \mathrm{~s}$ per level (from more detailed to less). In MongoDB, the total time to compute all aggregates was 3210 seconds with respectively $2611 \mathrm{~s}, 594 \mathrm{~s}, 5 \mathrm{~s}$ and $0.002 \mathrm{~s}$ per level (from more detailed to less). We can easily observe that computing the lower levels is much faster as the 
amount of data to be processed is smaller. The size of the aggregates (in terms of records) decreases too when we move down the hierarchy: 8.7 millions (level 2), 3.4 millions (level 3), 55 thousand (level 4) and 1 record in the bottom level.

\section{DISCUSSION}

In this section, we provide a discussion on our results. We want to answer three questions:

- Are the proposed models convincing?

- How can we explain performance differences across MongoDB and HBase?

- Is it recommended to use column-oriented and document-oriented approaches for OLAP systems and when?

The choice of our logical NoSQL models can be criticized for being simple. However, we argue that it is better to start from the simpler and most natural models before studying more complex ones. The two models we studied are simple and intuitive; making it easy to implement them. The effort to process the TPC-DS benchmark data was not difficult. Data from the TPC-DS benchmark was successfully mapped and inserted into MongoDB and HBase proving the simplicity and effectiveness of the approach.

HBase outperforms MongoDB with respect to data loading. This is not surprising. Other studies highlight the good performance on loading data for HBase. We should also consider that data fed to MongoDB was larger due to additional markup as MongoDB does not support csv-like files when the collection schema contains nested fields. Current benchmarks produce data in a columnar format (csv like). This gives an advantage to relational DBMS. The column-oriented model we propose is closer to the relational model with respect to the documentoriented model. This remains an advantage to HBase compared to MongoDB. We can observe that it becomes useful to have benchmarks that produce data that are adequate for the different NoSQL models.

At this stage, it is difficult to draw detailed recommendations with respect to the use of columnoriented or document-oriented approaches with respect to OLAP systems. We recommend HBase if data loading is the priority. HBase uses also less memory space and it is known for effective data compression (due to column redundancy). Computing aggregates takes a reasonable time for both and many aggregates take little memory space.
A major difference between the different NoSQL systems concerns interrogation. For queries that demand multiple attributes of a relation, the columnoriented approaches might take longer because data will not be available in one place. For some queries, the nested fields supported by document-oriented approaches can be an advantage while for others it would be a disadvantage. Studying differences with respect to interrogation is listed for future work.

\section{CONCLUSION}

This paper is about an investigation on the instantiation of OLAP systems through NoSQL approaches namely: column-oriented and documentoriented approaches. We have proposed respectively two NoSQL logical models for this purpose. The models are accompanied with rules that can transform a multi-dimensional conceptual model into a NoSQL logical model.

Experiments are carried with data from the TPCDS benchmark. We generate respectively datasets of size 1GB, 10GB and 100GB. The experimental setup show how we can instantiate OLAP systems with column-oriented and document-oriented databases respectively with HBase and MongoDB. This process includes data transformation, data loading and aggregate computation. The entire process allows us to compare the different approaches with each other.

We show how to compute an aggregate lattice. Results show that both NoSQL systems we considered perform well; with HBase being more efficient at some steps. Using map-reduce functions we compute the entire lattice. This is done for illustrative purposes and we acknowledge that it is not always necessary to compute the entire lattice. This kind of further optimizations is not the main goal of the paper.

The experiments confirm that data loading and aggregate computation is faster with HBase. However, document-based approaches have other advantages that remain to be thouroughly explored.

The use of NoSQL technologies for implementing OLAP systems is a promising research direction. At this stage, we focus on the modeling and loading stages. This research direction seems fertile and there remains a lot of unanswered questions.

Future work: We will list here some of the work we consider interesting for future work. A major issue concerns the study of NoSQL systems with respect to OLAP usage, i.e. interrogation for 
analysis purposes. We need to study the different types of queries and identify queries that benefit mostly for NoSQL models.

Finally, all approaches (relational models, NoSQL models) should be compared with each other in the context of OLAP systems. We can also consider different NoSQL logical ilmplementations. We have proposed simple models and we want to compare them with more complex and optimized ones.

In addition, we believe that it is timely to build benchmarks for OLAP systems that generalize to NoSQL systems. These benchmarks should account for data loading and database usage. Most existing benchmarks favor relational models.

\section{ACKNOWLEDGEMENTS}

These studies are supported by the ANRT funding under CIFRE-Capgemini partnership.

\section{REFERENCES}

Chaudhuri, S., Dayal, U., 1997. An overview of data warehousing and olap technology. SIGMOD Record, 26, ACM, pp. 65-74.

Colliat, G., 1996. Olap, relational, and multidimensional database systems. SIGMOD Record, 25(3), ACM, pp. 64-69.

Cuzzocrea, A., Bellatreche, L., Song, I.-Y., 2013. Data warehousing and olap over big data: Current challenges and future research directions. $16^{\text {th }}$ Int. Workshop on Data Warehousing and OLAP (DOLAP), ACM, pp. 67-70.

Dede, E., Govindaraju, M., Gunter, D., Canon, R. S., Ramakrishnan, L., 2013. Performance evaluation of a mongodb and hadoop platform for scientific data analysis. $4^{\text {th }}$ Workshop on Scientific Cloud Computing, ACM, pp. 13-20.

Dehdouh, K., Boussaid, O., Bentayeb, F., 2014. Columnar nosql star schema benchmark. Model and Data Engineering, LNCS 8748, Springer, pp. 281-288.

Golfarelli, M., Maio, D., and Rizzi, S., 1998. The dimensional fact model: A conceptual model for data warehouses. Int. Journal of Cooperative Information Systems, 7, pp. 215-247.

Gray, J., Bosworth, A., Layman, A., Pirahesh, H., 1996. Data Cube: A Relational Aggregation Operator Generalizing Group-By, Cross-Tab, and Sub-Total. Int. Conf. on Data Engineering (ICDE), IEEE Computer Society, pp. 152-159.

Han, D., Stroulia, E., 2012. A three-dimensional data model in hbase for large time-series dataset analysis. $6^{\text {th }}$ Int. Workshop on the Maintenance and Evolution of Service-Oriented and Cloud-Based Systems (MESOCA), IEEE, pages 47-56.

Jacobs, A., 2009. The pathologies of big data. Communications of the ACM, 52(8), pp. 36-44.

Kimball, R. Ross, M., 2013. The Data Warehouse Toolkit: The Definitive Guide to Dimensional Modeling. John Wiley \& Sons, Inc., $3^{\text {rd }}$ edition.

Lee, S., Kim, J., Moon, Y.-S., Lee, W., 2012. Efficient distributed parallel top-down computation of R-OLAP data cube using mapreduce. Int conf. on Data Warehousing and Knowledge Discovery (DaWaK), LNCS 7448, Springer, pp. 168-179.

$\mathrm{Li}, \mathrm{C}$., 2010. Transforming relational database into hbase: A case study. Int. Conf. on Software Engineering and Service Sciences (ICSESS), IEEE, pp. 683-687.

Malinowski, E., Zimányi, E., 2006. Hierarchies in a multidimensional model: From conceptual modeling to logical representation. Data and Knowledge Engineering, 59(2), Elsevier, pp. 348-377.

Morfonios, K., Konakas, S., Ioannidis, Y., Kotsis, N., 2007. R-OLAP implementations of the data cube. ACM Computing Survey, 39(4), p. 12.

Simitsis, A., Vassiliadis, P., Sellis, T., 2005. Optimizing etl processes in data warehouses. Int. Conf. on Data Engineering (ICDE), IEEE, pp. 564-575.

Ravat, F., Teste, O., Tournier, R., Zurfluh, G., 2008. Algebraic and Graphic Languages for OLAP Manipulations. Int. journal of Data Warehousing and Mining (ijDWM), 4(1), IGI Publishing, pp. 17-46.

Stonebraker, M., 2012. New opportunities for new sql. Communications of the ACM, 55(11), pp. 10-11.

Vajk, T., Feher, P., Fekete, K., Charaf, H., 2013. Denormalizing data into schema-free databases. $4^{\text {th }}$ Int. Conf. on Cognitive Infocommunications (CogInfoCom), IEEE, pp. 747-752.

Vassiliadis, P., Vagena, Z., Skiadopoulos, S., Karayannidis, N., 2000. ARKTOS: A Tool For Data Cleaning and Transformation in Data Warehouse Environments. IEEE Data Engineering Bulletin, 23(4), pp. 42-47.

TPC-DS, 2014. Transaction Processing Performance Council, Decision Support benchmark, version 1.3.0, http://www.tpc.org/tpcds/.

Wrembel, R., 2009. A survey of managing the evolution of data warehouses. Int. Journal of Data Warehousing and Mining (ijDWM), 5(2), IGI Publishing, pp. 24-56.

Zhao, H., Ye, X., 2014. A practice of tpc-ds multidimensional implementation on nosql database systems. $5^{\text {th }}$ TPC Tech. Conf. Performance Characterization and Benchmarking, LNCS 8391, Springer, pp. 93-108. 